\title{
A Robust Relative Positioning System for Multi-Robot Formations Leveraging an Extended GM-PHD Filter
}

\author{
Alicja Wasik, Alcherio Martinoli and Pedro U. Lima
}

\begin{abstract}
We propose a multi-robot tracking method to provide state estimates that allow a group of robots to maintain a formation even when the communication fails. We extend a Gaussian Mixture Probability Hypothesis Density filter to incorporate, firstly, absolute poses exchanged by the robots, and secondly, the geometry of the desired formation. Sensory detections, information about the formation, and communicated data are all combined in the extended Gaussian Mixture Probability Hypothesis Density filter. Our method is capable of maintaining the state estimates even when long-duration occlusions occur, and improves awareness of the situation when the communication rate is slow or sporadic. The method is evaluated using a high-fidelity simulator in scenarios with a formation of up to five robots. Experiments confirm the ability of the filter to deal with occlusions and refinement of the state estimate even when poses are exchanged at a low frequency, resulting in drastic reduction of the chance of collisions compared to a tracking-free implementation.
\end{abstract}

\section{INTRODUCTION}

Cooperative navigation is a critical feature for multi-robot systems. The ability to navigate in a formation enables a team of robots to perform activities not possible for single robots. To keep a desired formation geometry, it is necessary that each robot maintains a good estimate of the poses of the other robots, called the formation state. Since a robot role in the formation is usually associated to its unique identification number (ID), the robots must be capable of distinguishing each other. To realize an ID-based formation, multiple solutions have been proposed in the literature. In [1], robots perform teammate detection using combination of a LIDAR and a camera, where the camera also provides the identity of the neighboring robot. In [2], robots recognize themselves by extracting color blobs from a camera image. Both approaches aim at localizing a single local leader distinguishable by a marker. In [3], a follower vehicle maintains a formation with two leaders using acoustic ranging. The distinction between the leaders is ensured by an appropriate time-multiplexing scheme of the acoustic relative positioning signals. ID-dependent graphbased formation is achieved in [4]. Robots use a dedicated infrared range-and-bearing system and exchange messages containing robot IDs. In [5], agents in a formation localize

Supported by ISR/LARSyS Strategic Funds from FCT project FCT[UID/EEA/5009/2013] and FCT/11145/12/12/2014/S PhD grant.

Alicja Wasik and Pedro Lima are with the Institute for Systems and Robotics, Instituto Superior Técnico, Universidade de Lisboa, Portugal. alicja.wasikeepfl.ch and pedro.lima@tecnico.ulisboa.pt

Alicja Wasik and Alcherio Martinoli are with the Distributed Intelligent Systems and Algorithms Laboratory, School of Architecture, Civil and Environmental Engineering, École Polytechnique Fédérale de Lausanne, Switzerland. alcherio.martinolidepfl.ch using bearing-only measurements but are constrained to move with the motion type that is known by all agents a priori.

To the best of our knowledge, none of the existing multirobot tracking methods attempts to improve estimates of the robot poses based on specification of the desired formation shape or velocity. To obtain the states and the IDs, the robots either communicate with each other, or extract information from the sensors. We introduce a feedback loop between the formation controller and the state estimator by including knowledge about the desired formation geometry in the Gaussian Mixture Probability Hypothesis Density (GM-PHD) filter [6]. The use of such filter allows us to combine data from multiple information sources without the need to use heuristic methods for data association. Moreover, a GM-PHD filter does not fix the number of tracks a priori, therefore additional data regarding a target can be incorporated seamlessly [7].

The PHD filter estimates the number of targets and their states from a sequence of noisy measurements sets in the presence of detection uncertainties and false positives [6]. By representing approximation of the posterior density using Gaussian mixtures, the GM-PHD filter alleviates the computational intractability associated with the optimal multi-target Bayes filters. In spite of the multiple advantages, the GMPHD filter has a reduced ability to maintain track continuity when detections are missed. To overcome such difficulties, we propose to extend the GM-PHD with Formation Information resulting in the FI-GM-PHD filter. The FI-GM-PHD consists of two main components: i) the inception step incorporates poses of the robots exchanged via communication, when such information is available, ii) the coalition step integrates the expectation of the formation state based on the desired formation geometry. The expected formation state is either improving the current estimate or generating a new one, depending on the dissimilarity between the estimated formation state and the projected formation state.

This paper builds on our previous work on on-board relative localization system based on LIDAR measurements [8]. Our goal is to provide reliable robot pose estimates to be used when communication is of low rate or when it fails. For safety reasons, such backup system is necessary for establishing cooperative multi-robot navigation in humanpopulated environments [9], which is the long-term goal of this work. We consider ID-less [8] multi-robot tracking for formation control. It is important that the desired formation geometry is maintained, but since the robots are homogeneous, they can assume any role (target position). Roles are important to determine the range and the bearing that the robot has to maintain with respect to the other robots in the formation. 
Thus, for the estimates to be used in an formation control algorithm, a role assignment procedure finds a permutation that assigns the estimates to the roles. The problem of role assignment has been addressed previously using potential fields [10], market-based algorithm [11] and the Hungarian algorithm [12]. It was also applied in the context of graphbased formations [4]. While most of the works consider a static case, we assign the roles dynamically when the formation is navigating through the environment.

The main contribution of this work is to complete the ID-less method presented in [8] with a tracking component based on a FI-GM-PHD filter.

The paper is organized as follows. Section II reviews the GM-PHD filter and Section III introduces the FI-GMPHD filter. Section IV explains how the estimates obtained from the FI-GM-PHD are used in the formation control algorithm. Results from high-fidelity simulations are presented in Section V, followed by conclusions in Section VI.

\section{BACKGROUND}

Multi-object tracking methods estimate the states of multiple objects and their number from a sequence of multiple noisy measurements. The existing approaches include nonBayesian methods, such as Nearest Neighbor (NN) [13], Bayesian estimators, such as Multiple Hypothesis Tracking (MHT) [14] and statistics-based approaches such as Random Finite Set (RFS) theory [15]. Using RFSs is an optimal approach to multi-target tracking and a direct generalization of the single-target Bayes filter, but it is computationally intractable. A practical alternative is the Probability Hypothesis Density (PHD) filter, which propagates only the first-order statistical moment, called intensity, of the posterior multi-target state, but involves multiple integrals that have no closed solutions for the general case.

\section{A. The Gaussian Mixture PHD filter}

The GM-PHD filter [6] admits a closed form solution to the PHD recursion. Under linear, Gaussian assumptions on the target dynamics, the posterior intensity at time $k$ is a Gaussian mixture of the form:

$$
v_{k}(\mathbf{x})=\sum_{i=1}^{J_{k}} w_{k}^{(i)} \mathcal{N}\left(\mathbf{x} ; m_{k}^{(i)}, P_{k}^{(i)}\right)
$$

where each Gaussian component $i$ is associated with a weight $w_{k}^{(i)}, J_{k}$ is the number of Gaussian components representing the intensity and $\mathcal{N}(\cdot ; m, P)$ denotes a Gaussian density with mean $m$ and covariance $P$.

The GM-PHD filter involves four steps: 1) prediction, where the previous intensity evolves according to the motion model and where new targets can appear; 2) update, where the intensity is updated with the acquired measurements; 3) selection, including merging and pruning, to reduce the number of Gaussian components and; 4) state extraction.

1) Prediction: The predicted intensity at the time $k$ is a Gaussian mixture of the form:

$$
v_{k \mid k-1}(\mathbf{x})=v_{S, k \mid k-1}(\mathbf{x})+\gamma_{k}(\mathbf{x})
$$

where $v_{S, k \mid k-1}(\mathbf{x})$ is the survival intensity:

$$
v_{S, k \mid k-1}(\mathbf{x})=p_{S, k} \sum_{i=1}^{J_{k-1}} w_{k-1}^{(i)} \mathcal{N}\left(\mathbf{x} ; m_{k \mid k-1}^{(i)}, P_{k \mid k-1}^{(i)}\right)
$$

with $p_{S, k}$ being the probability of survival, and $\gamma_{k}(\mathbf{x})$ is the birth intensity with $J_{\gamma, k}$ components:

$$
\gamma_{k}(\mathbf{x})=\sum_{i=1}^{J_{\gamma, k}} w_{\gamma, k}^{(i)} \mathcal{N}\left(x ; m_{\gamma, k}^{(i)}, P_{\gamma, k}^{(i)}\right)
$$

The components of the survival intensity are computed from the previous intensity components according to a linear Gaussian motion model with $m_{k \mid k-1}^{(i)}=F_{k-1} m_{k-1}^{(i)}$ and $P_{k \mid k-1}^{(i)}=Q_{k-1}+F_{k-1} P_{k-1}^{(i)} F_{k-1}^{T}$ where $F_{k-1}$ is the state transition matrix and $Q_{k-1}$ is the process noise covariance. The mean values of the birth intensity components, $m_{\gamma, k}^{(i)}$, represent places, where new targets are likely to appear.

2) Update: Given a set of measurements $Z_{k}$, the posterior intensity is updated as follows:

$$
\begin{gathered}
v_{k}(\mathbf{x})=v_{T, k}(\mathbf{x})+\sum_{\mathbf{z} \in Z_{k}} v_{D, k}(\mathbf{x} ; \mathbf{z}) \\
v_{T, k}(\mathbf{x})=\sum_{i=1}^{J_{k \mid k-1}}\left(1-p_{D, k}\right) w_{k \mid k-1}^{(i)} \mathcal{N}\left(\mathbf{x} ; m_{k \mid k-1}^{(i)}, P_{k \mid k-1}^{(i)}\right) \\
v_{D, k}(\mathbf{x}, \mathbf{z})=\sum_{i=1}^{J_{k \mid k-1}} w_{k}^{(i)}(\mathbf{z}) \mathcal{N}\left(\mathbf{x} ; m_{k \mid k}^{(i)}(\mathbf{z}), P_{k \mid k}^{(i)}\right)
\end{gathered}
$$

where $p_{D, k}$ is the probability of detection. Intuitively, $v_{T, k}(\mathbf{x})$ is the missed-detection term, where the weight of each Gaussian component of the predicted intensity is discounted according to $p_{D, k}$. The $v_{D, k}(\mathbf{x} ; \mathbf{z})$ term, one for each measurement $\mathbf{z} \in Z_{k}$, is the detection term, which provides closed form expressions for computing the means, covariances and weights of $v_{k}$ from those of $v_{k \mid k-1}$ when a new set of measurements arrives. The equations for $w_{k}^{(i)}, m_{k \mid k}^{(i)}$ and $P_{k \mid k}^{(i)}$ depend on the observation matrix, $H_{k}$, the observation noise covariance $U_{k}$ and the expected clutter level, $\kappa_{k}(\mathbf{z})$. For the complete expressions of $w_{k}^{(i)}, m_{k \mid k}^{(i)}$ and $P_{k \mid k}^{(i)}$, please refer to [6].

3) Selection: To keep the problem tractable, components with weak weights are pruned: $I=\left\{i=1, \ldots, J_{k} \mid w_{k}^{(i)}>T\right\}$. All Gaussian components close to each other are merged into a single Gaussian. At first, a Gaussian component with the highest weight is selected $j=\operatorname{argmax}_{i \in I} w_{k}^{(i)}$. Then, all Gaussian components within the Mahalonobis distance $U$ from $j$ form a set $L=\left\{i \in I \mid\left(m_{k}^{(i)}-m_{k}^{(j)}\right)^{T}\left(P_{k}^{(i)}\right)^{-1}\left(m_{k}^{(i)}-m_{k}^{(j)}\right) \leq U\right\}$ of Gaussian components that are merged into one component:

$$
\begin{gathered}
\tilde{w}_{k}^{(l)}=\sum_{i \in L} w_{k}^{(i)}, \quad \tilde{m}_{k}^{(l)}=\frac{1}{\tilde{w}_{k}^{(l)}} \sum_{i \in L} w_{k}^{(i)} m_{k}^{(i)} \\
\tilde{P}_{k}^{(l)}=\frac{1}{\tilde{w}_{k}^{(l)}} \sum_{i \in L} w_{k}^{(i)}\left(P_{k}^{(i)}+\left(\tilde{m}_{k}^{(l)}-m_{k}^{(i)}\right)\left(\tilde{m}_{k}^{(l)}-m_{k}^{(i)}\right)^{T}\right)
\end{gathered}
$$

Finally, the number of Gaussian components is truncated to $J_{\max }$ components with the highest weights.

4) State extraction: The means of the Gaussian components are the local maxima of the posterior intensity $v_{k}$. Extraction of multi-target state estimates comes down to selection of the Gaussian means that have weights greater than a threshold $T_{S E}$. 


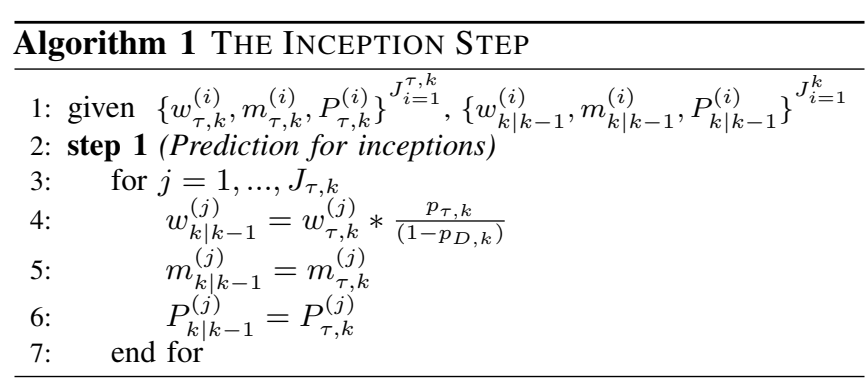

\section{GM-PHD FILTER WITH FORMATION INFORMATION}

The FI-GM-PHD filter consists of two steps, the inception step and the coalition step, for supplementing additional Gaussian components to the intensity.

\section{A. Inception of the Communicated Data}

Even when communication between the robots is possible, it may suffer of message losses, be of low rate or break occasionally. The inception step encodes the communicated data as a Gaussian mixture and adds it to the predicted intensity. The inception probability $p_{\tau, k}$ reflects the confidence about quality of the available communication and can be assessed online, based, for example, on the rate of incoming information. When communication is not available, $p_{\tau, k}=0$. The intensity of inception RFSs at time $k$ is a Gaussian mixture of the form:

$$
\tau_{k}(\mathbf{x})=\sum_{i=1}^{J_{\tau, k}} w_{\tau, k}^{(i)} \mathcal{N}\left(\mathbf{x} ; m_{\tau, k}^{(i)}, P_{\tau, k}^{(i)}\right)
$$

where $J_{\tau, k}$ is the number of Gaussian components, with the weight $w_{\tau, k}^{(i)}$, the mean $m_{\tau, k}^{(i)}$ and the covariance $P_{\tau, k}^{(i)}$. The new predicted intensity for time $k$ is modified from Eq. (2) and added as shown in Algorithm 1:

$$
v_{k \mid k-1}(\mathbf{x})=v_{S, k \mid k-1}(\mathbf{x})+\gamma_{k}(\mathbf{x})+p_{\tau, k} \tau_{k}(\mathbf{x})
$$

The inception intensity adds the hypothesis provided by the other robot, locally increasing the intensity analogously to the birth. The inception intensity is not suitable for the update step, because if only a fraction of the robots is exchanging the information, then performing the update would delete from the output the tracks of the robots that did not communicate.

Shaping of the inception RFSs requires a mapping between the state forming the intensity map and the communicated data. For example, if a state of the observed robot consists of its position and velocity, this information is to be extracted from the communicated message and encapsulated into $m_{\tau, k}^{(i)}$ of a single Gaussian component. The weight $w_{\tau, k}^{(i)}$ is the confidence of a target robot existing at $m_{\tau, k}^{(i)}$. Depending on the application, it can inform about the condition of the communication link, i.e. delays, message corruption etc. The covariance $P_{\tau, k}^{(i)}$ reflects the quality of the data, it is small for ground truth information and experimentally determined if the information is obtained from the self-localization system of the target robot. There is no need to associate the pose messages with the existing Gaussian components, as the PHD filter does not require further data association.

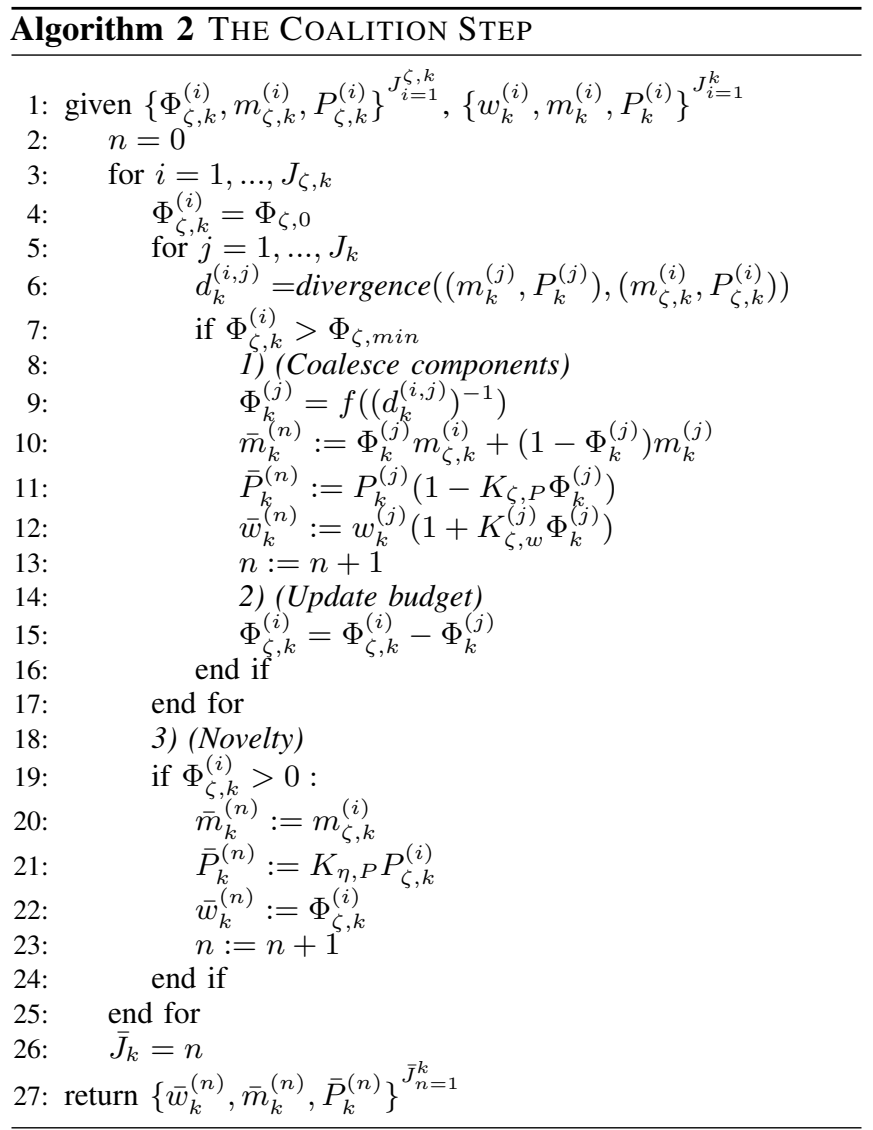

\section{B. The Expected Formation State}

Given the pose $\mathbf{x}_{i, k}=\left(x_{i, k}, y_{i, k}, \alpha_{i, k}\right)$ of the robot $R_{i}$, the expected position of the robot $R_{j}$ based on the desired formation geometry is:

$$
\left[\begin{array}{l}
h_{i j, k}^{x} \\
h_{i j, k}^{y}
\end{array}\right]=\left[\begin{array}{rr}
\cos \left(\alpha_{i, k}\right) & \sin \left(\alpha_{i, k}\right) \\
\sin \left(\alpha_{i, k}\right) & \cos \left(\alpha_{i, k}\right)
\end{array}\right]\left[\begin{array}{l}
b_{i j}^{x} \\
b_{i j}^{y}
\end{array}\right]+\left[\begin{array}{l}
x_{i, k} \\
y_{i, k}
\end{array}\right]
$$

where $b_{i j}$ is a bias, i.e. known desired spacing between the robot $R_{i}$ and $R_{j}$. The collection of the expected positions with respect to the robot $R_{i}$ of all the other robots in the formation is denoted by $\left\{h_{k}:=\left\{h_{i j, k}^{x}, h_{i j, k}^{y}\right\}, \mid j=1, \ldots, \Delta ; j \neq i\right\}$, where $\Delta$ is the number of robots in the formation and $\left|h_{k}\right|=\Delta-1$.

\section{Coalition of the Expected Formation States}

The coalition step extends the GM-PHD filter with an additional block, added after the update step. It combines the intensities obtained during the update step with the coalition intensity derived from the expected formation states. Thus, the Gaussian components constituting the coalition intensity serve as an outline of where the tracked robots are to be expected. The expected states $h_{k}$ are approximated using Gaussian mixture of the form:

$$
\zeta_{k}(\mathbf{x})=\sum_{i=1}^{J_{\zeta, k}} \Phi_{\zeta, k}^{(i)} \mathcal{N}\left(\mathbf{x} ; m_{\zeta, k}^{(i)}, P_{\zeta, k}^{(i)}\right)
$$

where $J_{\zeta, k}=\left|h_{k}\right|$ is the number of Gaussian components with the mean $m_{\zeta, k}^{(i)}$, the covariance $P_{\zeta, k}^{(i)}$ and the budget $\Phi_{\zeta, k}^{(i)}$. In particular, the mean intensity is $m_{\zeta, k}=\left[\begin{array}{lll}h_{k}^{x} & h_{k}^{y}, & 0,0\end{array}\right]^{T}$. 


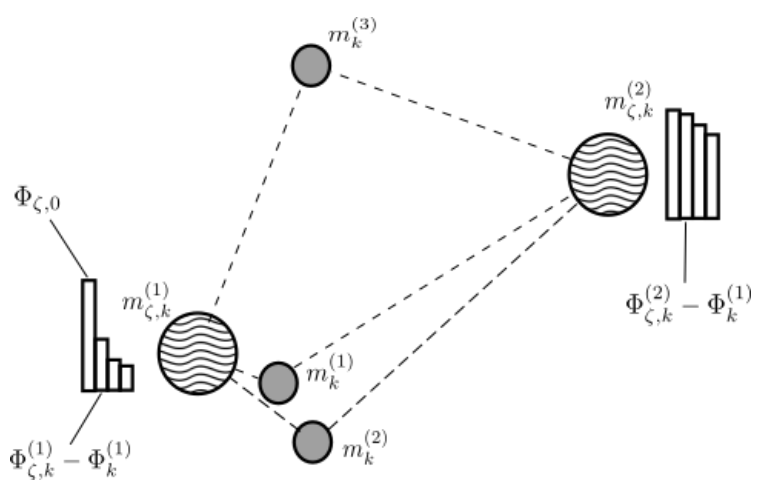

Fig. 1: Illustration of the coalition step. Three posterior intensity components, $m_{k}^{(1)}, m_{k}^{(2)}, m_{k}^{(3)}$ are compared against two coalition components, $m_{\zeta, k}^{(1)}, m_{\zeta, k}^{(2)}$ with the corresponding budgets $\Phi_{\zeta, k}^{(1)}, \Phi_{\zeta, k}^{(2)}$. Closeness of the $m_{\zeta, k}^{(1)}$ to the $m_{k}^{(1)}$ decreases significantly its budget, while for $m_{\zeta, k}^{(2)}$, the budget does not become depleted and a novelty is created.

The coalition step is detailed in Algorithm 2. Each component $i=1, \ldots, J_{\zeta, k}$ of the coalition intensity (12) at time $k$ is given an initial budget $\Phi_{\zeta, k}^{(h)}=\Phi_{\zeta, 0}$. Then, all the components forming the posterior intensity $v_{k}(\mathbf{x}), j=1, \ldots, J_{k}$, are compared against $i$, using some measure of divergence. If the distance is small, the possibility that both components correspond to the same target is high and the components $i$ and $j$ are coalesced to form a new Gaussian component, with a mean of intensity being a combination of the means of the two, the covariance and the weight being the covariance and the weight of $j$ modified as a function of the divergence. If the distance between $i$ and $j$ is large, the likeliness that $j$ is associated with $i$ is small and $j$ is propagated further with little change. This diverse behavior is assured by using a sigmoid-shaped function of the divergence when comparing the components. The duality of the function also ascertains that the condition in Line 7 of Algorithm 2 does not depend on the order in which the Gaussian components are evaluated. The budget of the coalition component is decreased with every posterior component that has been found close to it, and has two major advantages. Firstly, it limits the number of new components that can originate around it. Secondly, a budget left at the end of iteration indicates that one of the coalition components did not have a corresponding component in the posterior, whether because of the missed detection, occlusion, or field of view limits. In that case, a new component, called the novelty is created with the mean at the area where a robot is expected to be.

As an example, consider three posterior intensity components, $m_{k}^{(1)}, m_{k}^{(2)}, m_{k}^{(3)}$ compared against two coalition components, $m_{\zeta, k}^{(1)}, m_{\zeta, k}^{(2)}$ with the corresponding budgets $\Phi_{\zeta, k}^{(1)}, \Phi_{\zeta, k}^{(2)}$ initially set to $\Phi_{\zeta, 0}$ (see Fig. 1). Closeness of the $m_{k}^{(1)}$ to the $m_{\zeta, k}^{(1)}$ decreases significantly its budget $\Phi_{\zeta, k}^{(1)}$, and a new component is created as a combination of the two. For $m_{\zeta, k}^{(2)}$, the budget does not become depleted and a novelty is created. Finally, component $m_{k}^{(3)}$ has no close correspondence in $\zeta_{k}$ and it is propagated with no modification.

The coalition step and the inception step supplement the
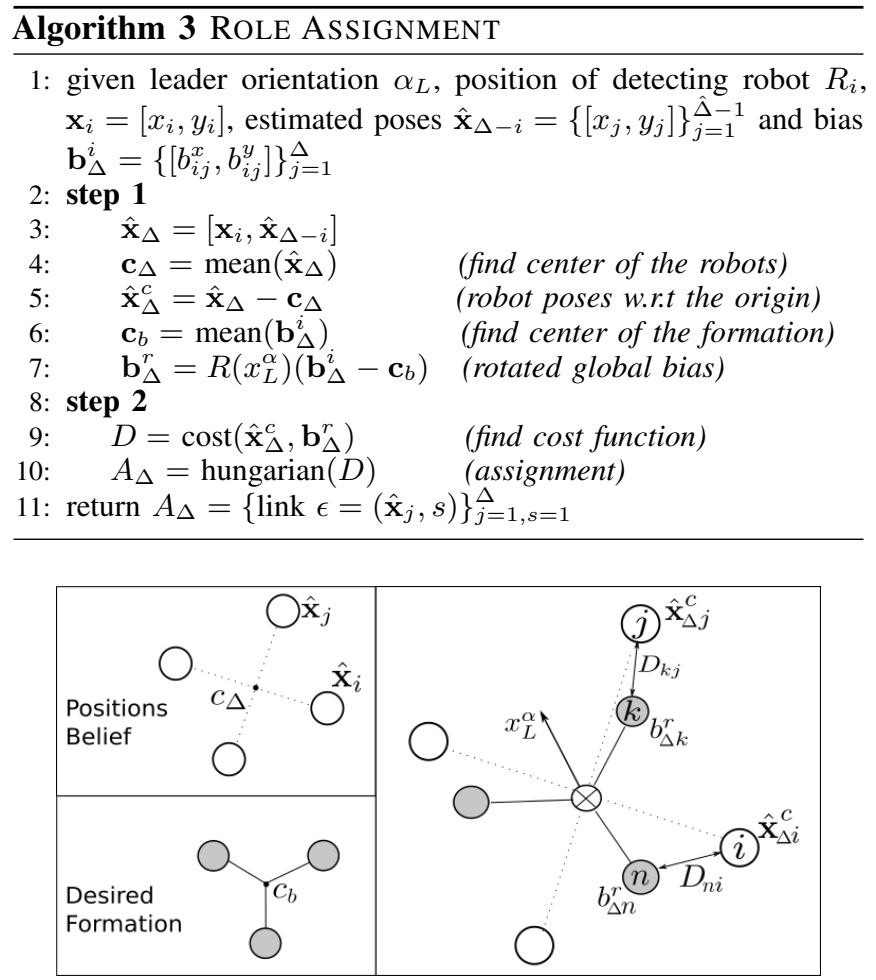

Fig. 2: Illustration of the role assignment step. Center of the estimates $\mathbf{c}_{\Delta}$ and center of the formation $\mathbf{c}_{b}$ are brought to a common reference frame at the origin, where the translated estimates are $\hat{\mathbf{x}}_{\Delta}^{c}$, and the bias, translated and rotated according the leader orientation $x_{L}^{\alpha}$, is $\mathbf{b}_{\Delta}^{r} . D_{n i}$ is the cost of associating bias $\mathbf{b}_{\Delta n}^{r}$ with estimate $\hat{\mathbf{x}}_{\Delta i}^{c}$.

intensity with additional Gaussian components. In the case the robot has not been tracked, adding a new Gaussian component creates a new target based on the high probability that the target is there. This is analogous to the birth process of the GM-PHD filter [6]. In the case the robot has already been tracked, adding a new Gaussian component that corresponds to that robot increases the likelihood of the robot being present at that position, provided that the state extraction takes into account the fact that multiple targets cannot occupy the same physical position, i.e. the components with weight above one are extracted as single targets.

\section{Multi-Robot tracking FOR Formation CONTROL}

The multi-target state estimates of the FI-GM-PHD filter, after being assigned to the roles, provide the range and bearing information in the graph-based formation algorithm.

\section{A. Graph-Based Formation Control}

Details of the formation control algorithm can be found in [9]. The formation is comprised of $\Delta$ holonomic following robots and one centralized virtual leader, which is a point on a predefined trajectory. Such information can be communicated a priori or during execution to all robots. The pose of the virtual leader is $\mathbf{x}_{L}=\left(x_{L}, y_{L}, \alpha_{L}\right)$, while of the following robot $R_{i}$ is $\mathbf{x}_{i}=\left(x_{i}, y_{i}, \alpha_{i}\right)$. For a robot $R_{i}$, the formation control is achieved as: 


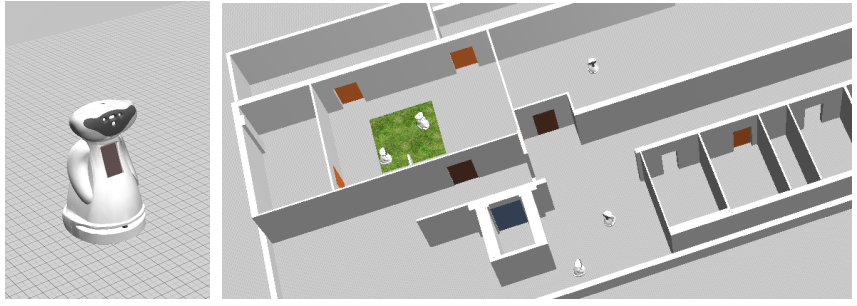

Fig. 3: A screenshot from the Webots simulator. The MBot robot (left) and the simulated indoor environment (right).

$$
\begin{aligned}
& \dot{x}_{i}=\frac{1}{\Delta_{i}^{L}+1} \sum_{j=1}^{\Delta_{i}^{L}}\left[-\mathcal{L}_{i j}\left(r_{i j}(t) \cos \left(\gamma_{i j}(t)\right)-b_{i j}^{x}(t)\right)\right] \\
& \dot{y}_{i}=\frac{1}{\Delta_{i}^{L}+1} \sum_{j=1}^{\Delta_{i}^{L}}\left[-\mathcal{L}_{i j}\left(r_{i j}(t) \sin \left(\gamma_{i j}(t)\right)-b_{i j}^{y}(t)\right)\right]
\end{aligned}
$$

where $\Delta_{i}^{L}$ is the number of neighbors of robot $R_{i}$ including the leader, $\mathcal{L}$ is a non-stationary Laplacian, $r_{i j}$ and $\gamma_{i j}$ are the Euclidean relative position and the bearing between the robots $R_{i}$ and $R_{j}$ respectively. The bias vectors $\mathbf{b}_{i}^{x}, \mathbf{b}_{i}^{y} \in \mathbb{R}^{\Delta_{i}^{L}}$ define the desired robot-leader and inter-robot distances. Finally, the robots are omnidirectional, so the follower's heading is controlled so as to match the orientation of the leader, $\dot{\alpha}_{i}=\alpha_{L}-\alpha_{i}$.

\section{B. Role Assignment}

The role assignment procedure finds a permutation that assigns the estimates to the target positions in the formation. Although we use the graph-based framework notation, the method can be generalized to other formation algorithms. The procedure is sketched in Algorithm 3. Consider a formation with its geometry uniquely determined by a bias matrix $\mathbf{b}_{\Delta}$, a set of desired inter-robot distances. The heading of the formation is tangential to the formation trajectory, assumed to be known by all the robots. In our case the heading is established by the virtual leader, $\alpha_{L}$. The bias and the estimates are brought to a common reference frame by matching the center of the rotated global bias $\mathbf{b}_{\Delta}^{r}$ with the center of the estimates $\hat{\mathbf{x}}_{\Delta}^{c}$ (see Fig. 2). The combination of the estimates and the roles resulting in the smallest cost is computed using the Hungarian algorithm [16]. The role assignment procedure, run by the robot $R_{i}$, provides a set of links, $A_{\Delta}=\left\{\epsilon=\left(\hat{\mathbf{x}}_{i}, s\right),\right\}_{s=1}^{\Delta}$. The link $\epsilon=\left(\hat{\mathbf{x}}_{j}, s\right)$ is used for correct matching of the bias $b_{i s}(t)$ with $r_{i j}(t)$ and $\gamma_{i j}(t)$ in Eq. 13, while its cost gives the confidence about the assignment. More precisely, estimate $\hat{\mathbf{x}}_{j}$ is associated with role " $s$ " and its subscript is changed to $\hat{\mathbf{x}}_{s}$, with the range $r_{i s}$ and the bearing $\gamma_{i s}$ with respect to the robot $R_{i}$. The estimate $\hat{\mathbf{x}}_{s}$ is coupled with bias $b_{i s}$ that corresponds to the " $s$ th" place in the formation.

\section{EXPERIMENTS}

Experiments have been performed in the high-fidelity simulator Webots [17], with holonomic MBot robots of diameter $0.65 \mathrm{~m}$ shown in Fig. 3. The robots are equipped with two LIDARs providing $360^{\circ}$ field of view and sensing range $4 \mathrm{~m}$, which are accurately simulated and calibrated using real data. Robots self-localize using the $\mathrm{AMCL}^{1}$

${ }^{1} \mathrm{AMCL}$ (http://wiki.ros.org/amcl)

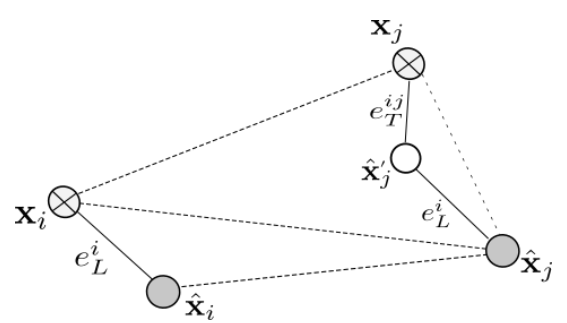

Fig. 4: The measures used for evaluating the tracking performance. The ground truth pose of the target is $\mathbf{x}_{j}$ and its estimated pose is $\hat{\mathbf{x}}_{j}$. The absolute self-localization error $e_{L}^{i}$ of the detecting robot $R_{i}$ is subtracted at $\hat{\mathbf{x}}_{j}^{\prime}$, to result in the tracking error $e_{T}^{i j}$.

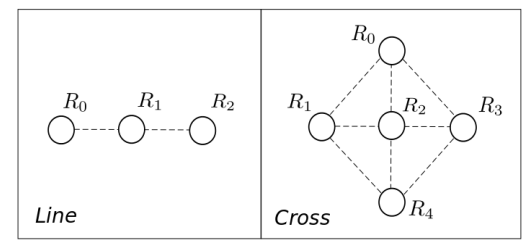

Fig. 5: The formation geometries used in the experiments.

package from ROS, with the accuracy of approximately $10 \mathrm{~cm}$. For robot detection we use an on-board relative localization system originally presented in [8]. The measurement is a position returned in the form $\mathbf{z}_{i}=\left[z_{i}^{x}, z_{i}^{y}\right]^{T}$.

The state $\mathbf{x}_{j}=\left(x_{j}, y_{j}, \dot{x}_{j}, \dot{y}_{j}\right)$ of each target in the global reference frame consists of position and velocity. Each target has the survival probability $p_{S, k}=0.95$, and follows the linear Gaussian dynamical model with:

$$
F_{k}=\left[\begin{array}{rr}
I_{2} & \delta I_{2} \\
0_{2} & I_{2}
\end{array}\right], \quad Q_{k}=\sigma_{v}^{2}\left[\begin{array}{cc}
\frac{\delta^{4}}{4} I_{2} & \frac{\delta^{3}}{2} I_{2} \\
\frac{\delta^{3}}{2} I_{2} & \delta^{2} I_{2}
\end{array}\right]
$$

where $I_{n}$ and $0_{n}$ denote, respectively, the $n \times n$ identity and zero matrices, $\delta=0.1 s$ is the time step, and $\sigma_{v}^{2}=2.0$ is the standard deviation of the process noise. The measurement $\mathbf{z}_{i}=\left[z_{i}^{x}, z_{i}^{y}\right]^{T}$ is a noisy version of the position and follows a linear Gaussian observation model with $H_{k}=\left[I_{2}, 0_{2}\right]$, $U_{k}=\sigma_{\epsilon}^{2} I_{2}$, where $\sigma_{\epsilon}^{2}=0.2 \mathrm{~m}$ is the standard deviation of the measurement noise, determined empirically.

At initialization, the filter run by robot $R_{i}$ is supplied with a birth RFS in the vicinity of the initial formation state with $m_{\gamma, 0}^{(j)}=\left[h_{i j, 0}^{x}, h_{i j, 0}^{y}, 0,0\right]^{T}, j=1, \ldots,\left|h_{k}\right|$ and $P_{\gamma, 0}=\operatorname{diag}\left([1.6,1.6,1.9,1.9]^{T}\right)$. The parameters for merging and pruning are: $J_{\max }=10, U=0.5, T=10^{-4}$ and we select $\Delta-1$ highest Gaussian components above $T_{S E}=0.7$. Poisson distributed clutter level is $\kappa_{k}(z)=1$.

The coalition mean intensity $m_{\zeta, k}^{(j)}=\left[\begin{array}{llll}h_{i j, k}^{x} & h_{i j, k}^{y}, 0,0\end{array}\right]^{T}$, $j=1, \ldots, J_{\zeta, k}$ is based on the expected formation state, the covariance is $P_{\zeta, k}^{(j)}=\operatorname{diag}\left([0.05,0.05,0.1,0.1]^{T}\right)$ and the budget $\Theta_{\zeta, k}^{(j)}=\Theta_{\zeta, 0}$, where $\Theta_{\zeta, 0}=1.0$. The function used for coalescing the components is $f(x)=\frac{1}{1+\exp (-20(x-0.85))}$. The other parameters are $K_{\zeta, P}=0.1, K_{\eta, P}=10, K_{\zeta, w}^{(j)}=\max \left(1.0, \frac{1}{w_{k}^{(j)}}\right), \Theta_{\tau, \min }=-0.01$.

In the experiments, when communication is available, the inception probability is $p_{\tau, k}=1.0$, and the mean inception RFSs $m_{\tau, k}^{(j)}=\left[x_{j, k}^{x}, x_{j, k}^{y}, 0,0\right]^{T}$ is generated based on the position $\overline{\mathbf{x}}_{j, k}=\left[x_{j, k}, y_{j, k}\right]$ shared by the robot $R_{j}$. The covariance is $P_{\tau, k}=\operatorname{diag}\left([0.01,0.01,0.02,0.02]^{T}\right)$ 
and the weight $w_{\tau, k}=0.7$. To reduce the impact of the coalition intensity when communicated data is available, $P_{\zeta, k}=\operatorname{diag}\left([0.5,0.5,1.0,1.0]^{T}\right)$.

\section{A. Performance Evaluation}

The tracking performance measure is the difference between the ground truth pose $\mathbf{x}_{j}$ of the target and its estimated pose $\hat{\mathbf{x}}_{j}$, corrected by taking into account the absolute selflocalization error $e_{L}^{i}$ of the detecting robot $R_{i}$ (see Fig. 4):

$$
\mathbf{e}_{T}^{i j}=\left\|\hat{\mathbf{x}}_{j}-\mathbf{x}_{j}\right\|-e_{L}^{i}
$$

The formation error is evaluated using an average difference between the desired distances between the robots and the actual distances:

\section{B. Scenarios}

$$
e_{D}=\frac{2}{\Delta(\Delta-1)} \sum_{i=1}^{\Delta} \sum_{j=i}^{\Delta}\left|\left\|\mathbf{x}_{i}-\mathbf{x}_{j}\right\|-\left\|b_{i j}^{x y}\right\|\right|
$$

The aim of the following scenarios is 1) to show the advantage of the FI-GM-PHD filter when long-term occlusions occur; 2) to use the obtained position estimates in a closed loop with the formation controller; 3) to test fusion of the information from tracking and communication when the latter is unreliable. The pose of the virtual leader is evolved by a central node and communicated to all robots every time step. In each experiment we perform 10 sequential runs.

1) Open-Loop Tracking: The dataset has been collected while three robots follow the virtual leader on a figure eight trajectory in a line formation (see Fig. 5). Each run lasts $110 \mathrm{~s}$. The same dataset is used to evaluate and compare the standard GM-PHD filter and the FI-GM-PHD filter. The formation is maintained using self-localization poses communicated between the robots.

2) Tracking for Formation Control: Five robots $R_{0}-R_{4}$ forming a cross shape with a bias $b_{12}^{x}=0.8$ (see Fig. 5) move on an ellipsoidal trajectory around a wall. The robots $R_{3}$ and $R_{4}$ use solely the estimated positions, while $R_{0}, R_{1}$ and $R_{2}$ use the communicated positions. Each run lasts $180 \mathrm{~s}$.

3) Tracking with Limited Communication: In the first experiment (Case A) the robots move in a formation using positions communicated at a low frequency of $10 \delta(1 \mathrm{~s})$. In the second experiment (Case B) the communicated information is fed into the the FI-GM-PHD tracker upon reception (every $10 \delta$ ) using the inception step. Detection and tracking runs every time step $\delta$. The five robots $R_{0}-R_{4}$ form a cross shape with a bias $b_{12}^{x}=1.0$. Each run lasts $180 \mathrm{~s}$.

\section{Results}

1) Open-Loop Tracking: Fig. 6 (Left) shows the trajectories of the robots and the position estimates obtained by the detecting robot $R_{0}$ from the standard GM-PHD tracker. The GM-PHD filter does not have a mechanism that could deal with missed detections over a long duration: due to occlusion of robot $R_{2}$, in this scenario $R_{0}$ is only capable of maintaining the track of robot $R_{1}$ (in yellow). The GM-PHD filter is also sensitive to appearance of clutter caused by distorted detections (Fig. 7, e.g. $t=20 s$ ). The FI-GM-PHD method (Fig. 6 right) maintains the tracks of both robots $R_{1}$ and $R_{2}$,
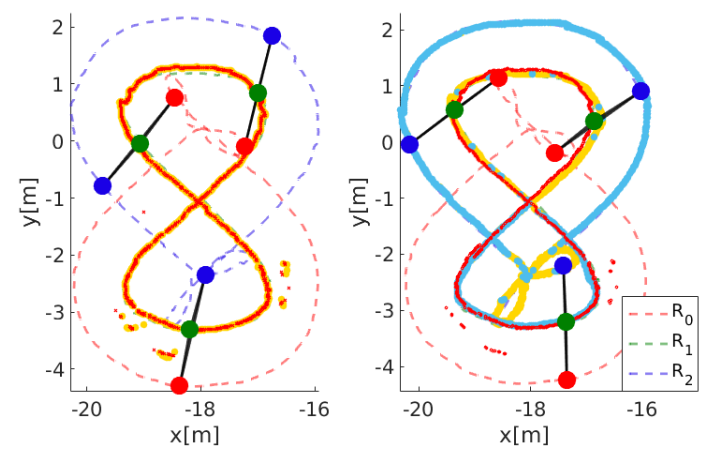

Fig. 6: Scenario 1. Trajectories of the robots, measurements (red) and estimates (yellow and cyan). (Left) Standard GM-PHD filter. (Right) FI-GM-PHD filter.
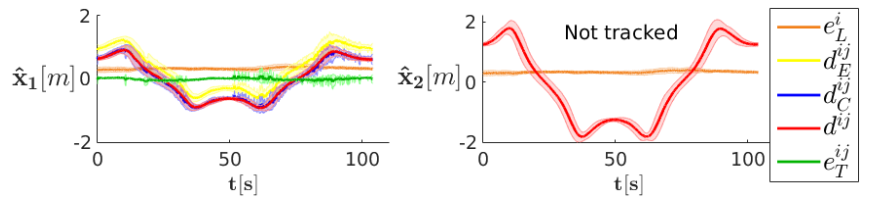

Fig. 7: Scenario 1. Tracking error of the GM-PHD filter in the x-dimension. The self-localization error $e_{L}^{i}$ of $\mathrm{R}_{i}$ is in brown, the error of tracking the robot $\mathbf{R}_{j}, e_{T}^{i j}$, is in green. $d^{i j}$ is the ground truth distance between $\mathbf{R}_{i}$ and $\mathbf{R}_{j}, d_{E}^{i j}$ is the distance between $\mathbf{R}_{i}$ and its estimate of $\mathbf{R}_{j}, d_{C}^{i j}$ is $d_{E}^{i j}$ corrected by subtracting the self-localization error. The lack of plots for $\hat{\mathbf{x}}_{2}$ indicates absence of the track and the tracking error is infinite.
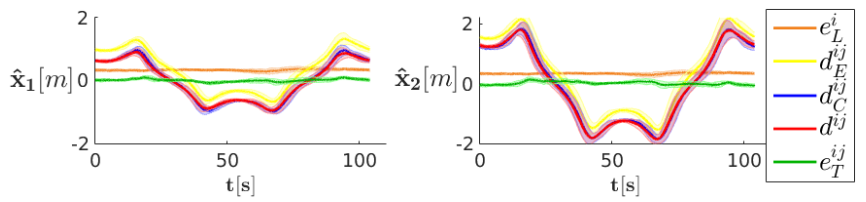

Fig. 8: Scenario 1. Tracking error of the FI-GM-PHD filter in the x-dimension. The tracking error $e_{T}^{i j}$ is in green.

and reduces impact of the clutter (Fig. 8). For the detected tracks, both methods have similar average error of $1 \mathrm{~cm}$ mean and variance of up to $8 \mathrm{~cm}$.

2) Tracking for Formation Control: Fig. 9 (top) shows the trajectories, where $R_{3}$ and $R_{4}$ use solely the estimates provided by the FI-GM-PHD tracker. Although the formation error is within acceptable bounds, lower than half the robot diameter (see Fig. 9 bottom), we observe that certain conditions may cause a small drift of the robot (e.g., robot $R_{4}$ ). Even small inaccuracies in the formation shape caused by using estimates in place of the actual states, with time can increase the misprint of the expected formation state. Sharp turns can cause inconsistency in the role assignment and when five robots are involved, occlusions occur in abundance. Nevertheless, during re-assignment of the coalition intensity budget, the FI-GM-PHD method puts a high emphasis on all the detections, therefore the expectation of the formation state is subject to continuous correction and the robots can recover.

3) Tracking with Limited Communication: In Case A, the shape of the formation becomes distorted and the robots tend to oscillate around their desired positions in the formation, 

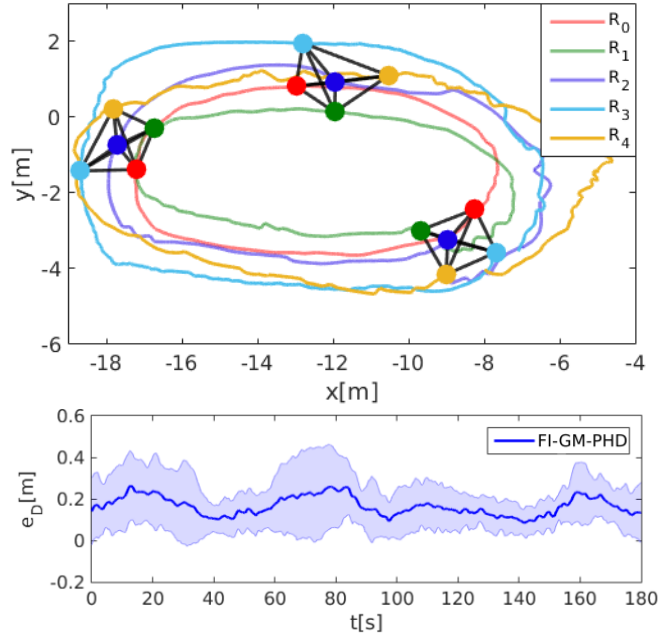

Fig. 9: Scenario 2. (Top) Trajectories of the robots. Robots $R_{3}$ and $R_{4}$ use estimates of the FI-GM-PHD tracker to maintain the formation. (Bottom) The formation error.

because the corrective control actions use information from up to $10 \delta$ earlier. A number of runs (not included in the calculation of $e_{D}$ ) failed to fully execute because of robot collisions. Collisions occurred in approximately $30 \%$ of all the commenced runs. In Case B, the formation maintains close-todesirable shape with the error $12 \%$ lower than in Case A (see Fig. 10). No collisions have been recorded and the system displays stable performance during 10 sequential runs. By complementing the communicated data, the FI-GM-PHD filter maintains the tracks between the arrivals of the communicated data. While in Case A the state information is updated only $10 \%$ of the time, the FI-GM-PHD filter maintains the tracks for on average $82 \%$ of the time, although track maintenance depends on the chosen parameters, especially the detection probability, which trades the ability to maintain the track for the accuracy and clutter handling.

\section{CONCLUSIONS}

This paper presented a method for multi-robot tracking for formation control. The FI-GM-PHD filter provided reliable and robust state estimates, obtained as a consequence of closing the loop between the formation and the tracking system. The experimental results confirmed the ability of the filter to deal with abundance of occlusions and clutter. When the communication was sparse, the FI-GM-PHD tracker provided reliable state estimates that not only lowered the formation error, but also drastically reduced the chance of collisions compared to a control without the tracking layer.

The FI-GM-PHD tracker will be validated with real robots. Our intention is to use the proposed method for our reconfigurable robot formations, with the final goal of deploying a team of cooperative robots in environments populated with humans. The FI-GM-PHD tracker is to serve as a backup system for the situations, when the robots temporarily loose their ability to communicate. Limitations of the method will be further studied, focusing on the performance in situations initialized by a spatial configuration that is far off from the desired formation geometry. Moreover, in case the formation

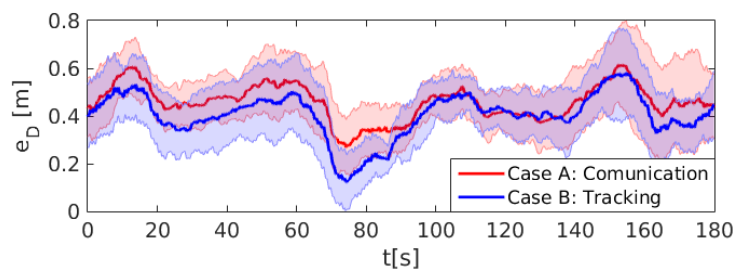

Fig. 10: Scenario 3. The formation error for the low-rate communicated information and the FI-GM-PHD filter.

breaks, we intend to develop a behavior-based plan that would allow the robots to recalculate the expected formation state to continue taking full advantage of the FI-GM-PHD tracker.

\section{REFERENCES}

[1] J. Fredslund and M. J. Matarić, "A general algorithm for robot formations using local sensing and minimal communication," IEEE Trans. on Robotics and Automation, vol. 18, no. 5, pp. 837-846, 2002.

[2] R. Alur, A. Das, J. Esposito, R. Fierro, G. Grudic, Y. Hur, V. Kumar, I. Lee, J. P. Ostrowski, G. Pappas, B. Southall, J. Spletzer, and C. J. Taylor, A Framework and Architecture for Multirobot Coordination, pp. 303-312. Berlin, Heidelberg: Springer Berlin Heidelberg, 2001.

[3] J. M. Soares, A. P. Aguiar, A. M. Pascoal, and A. Martinoli, "Joint ASV/AUV range-based formation control: theory and experimental results," in IEEE Int. Conf. on Robotics and Automation, pp. 5579-5585, 2013.

[4] R. Falconi, S. Gowal, and A. Martinoli, "Graph-based distributed control of non-holonomic vehicles endowed with local positioning information engaged in escorting missions," in IEEE Int. Conf. on Robotics and Automation, pp. 3207-3214, 2010.

[5] M. Ye, B. D. O. Anderson, and C. Yu, "Bearing-only measurement self-localization, velocity consensus and formation control," IEEE Transactions on Aerospace and Electronic Systems, vol. 53, pp. 575586, April 2017.

[6] B.-N. Vo and W.-K. Ma, "The gaussian mixture probability hypothesis density filter," IEEE Trans. on Signal Processing, vol. 54, no. 11, pp. 4091-4104, 2006.

[7] M. Vasic and A. Martinoli, "A collaborative sensor fusion algorithm for multi-object tracking using a gaussian mixture probability hypothesis density filter," Proc. of the 2015 IEEE 18th Int. Conf. on Intelligent Transportation Systems, pp. 491-498, 2015.

[8] A. Wasik, R. Ventura, J. N. Pereira, P. U. Lima, and A. Martinoli, "Lidar-based relative position estimation and tracking for multi-robot systems," in Robot 2015: Second Iberian Robotics Conf., pp. 3-16, 2016.

[9] A. Wasik, J. N. Pereira, R. Ventura, P. U. Lima, and A. Martinoli, "Graph-based distributed control for adaptive multi-robot patrolling using local formation transformation," in IEEE Int. Conf. on Intelligent Robots and Systems, pp. 1721-1728, 2016.

[10] M. M. Zavlanos and G. J. Pappas, "Dynamic assignment in distributed motion planning with local coordination," IEEE Trans. on Robotics, vol. 24, no. 1, pp. 232-242, 2008.

[11] N. Michael, M. M. Zavlanos, V. Kumar, and G. J. Pappas, "Distributed multi-robot task assignment and formation control," in IEEE Int. Conf. on Robotics and Automation, pp. 128-133, 2008.

[12] Z. Sun and Y. Xia, "Consensus-based formation control with dynamic role assignment," in The 26th Chinese Control and Decision Conf., pp. 3681-3686, 2014.

[13] S. S. Blackman, "Multiple-target tracking with radar applications," Dedham, MA, Artech House, Inc., vol. 1, 1986.

[14] S. Blackman and R. Popoli, Design and Analysis of Modern Tracking Systems. Artech House Radar Library, Artech House, 1999.

[15] R. P. Mahler, "Multitarget Bayes filtering via first-order multitarget moments,' IEEE Trans. on Aerospace and Electronic Systems, vol. 39, no. 4, pp. 1152-1178, 2003.

[16] H. W. Kuhn, "The hungarian method for the assignment problem," Naval Research Logistics Quarterly, vol. 2, no. 1-2, pp. 83-97, 1955.

[17] O. Michel, "Cyberbotics ltd. webots: professional mobile robot simulation," Int. Journal of Advanced Robotic Systems, vol. 1, no. 1, p. 5, 2004. 